\title{
Implementação da monitoria remota em anestesiologia durante a pandemia da COVID-19: interface, contribuições e aprendizado
}

Implementation of remote mentoring in anesthesiology during the pandemic of COVID-19: interface, contributions and learning

Implementación de la monitoria remota en anestesiología durante la pandemia COVID-19: interfaz, contribuciones y aprendizaje

Marco Antonio Cardoso Resende ${ }^{1 *}$, Rogerio Luiz da Rocha Videira1, Ana Carolina Fortes Bastos da Costa ${ }^{1}$, Laís Sales Freire Silva ${ }^{1}$, Manoel Fernando de Oliveira Rodrigues ${ }^{1}$, Alexandra Rezende Assad ${ }^{1}$.

\section{RESUMO}

Objetivo: Descrever a implementação da monitoria online em módulo de anestesiologia, com uso de tecnologia da informação e comunicação por meio de atividades remotas durante a pandemia de COVID - 19 . Relato de experiência: Trata-se de estudo descritivo na modalidade relato de experiência com vivências de docentes e discentes de medicina de uma universidade no estado do Rio de Janeiro, após a interrupção das aulas presenciais, e seu impacto no processo de ensino-aprendizagem. A dinâmica metodológica contemplou atividades assíncronas, com postagem de artigos, capítulos de livros, estudo dirigido, aulas gravadas no Google Sala de Aula, vídeos curtos de procedimentos, e síncronas online na semana seguinte com cada tema. Como forma de registrar as percepções e repensar atividades futuras foi aplicado questionário semiestruturado para professores e alunos. As expectativas pedagógicas da disciplina e interação com os alunos foram atendidas plenamente de acordo com cinco professores e parcialmente por quatro, enquanto $78,9 \%$ dos alunos tiveram suas expectativas atendidas. Considerações finais: Apesar de nenhum dispositivo tecnológico ser capaz de suprir a necessidade de interação presencial no ensino médico, a monitoria online foi fundamental por atuar como facilitadora, estabelecendo pontes na interface docente-discente.

Palavras-chave: Anestesiologia, Educação, Covid-19, Monitoria.

\section{ABSTRACT}

Objective: To describe the implementation of online mentoring in an anesthesiology module, using information and communication technology through remote activities during the COVID-19 pandemic. Experience report: This is a descriptive study in the form of an experience report with the experiences of teachers and medical students of an university in Rio de Janeiro, after the interruption of classroom lectures, and its impact on the teaching-learning process. The methodological dynamics included asynchronous activities, with posting of articles, book chapters, directed study, classes recorded on Google Classroom, short videos of procedures, and online synchronous activities in the following week with each theme. As a way of recording perceptions and rethinking future activities, a semi-structured questionnaire was applied to teachers and students. The pedagogical expectations of the course and interaction with students were fully met by five professors and partially met by four, while $78.9 \%$ of the students had their expectations met. Final considerations: Although no technological device is capable of meeting the need forface-to-face interaction in medical education, online mentoring was fundamental for acting as a facilitator, establishing bridges in the teacher-student interface.

Keywords: Anesthesiology, Education, Covid-19, Mentoring.

1 Universidade Federal Fluminense (UFF), Niterói - RJ. *E-mail: macresende@gmail.com 


\begin{abstract}
RESUMEN
Objetivo: Describir la implementación de la monitoría remota en anestesiología, con el uso de la tecnología informática y de la comunicación a través de actividades a distancia durante la pandemia COVID -19. Informe de experiencia: Se trata de un estudio descriptivo en forma de informe de experiencia con las vivencias de profesores y estudiantes de medicina de una universidad de Rio de Janeiro, tras la interrupción de las clases, y su impacto en el proceso de enseñanza-aprendizaje. La dinámica metodológica incluyó actividades asincrónicas, con publicación de artículos, capítulos de libros, estudio dirigido, clases grabadas en Go ogle Classroom, videos cortos de procedimientos y actividades sincrónicas en línea a la semana siguiente con cada tema. Para registrar las percepciones y replantear las actividades futuras, se administró un cuestionario semiestructurado a profesores y alumnos. Las expectativas pedagógicas del curso y la interacción con los alumnos fueron totalmente satisfechas por cinco profesores y parcialmente por cuatro, mientras que el $78,9 \%$ de los alumnos vieron satisfechas sus expectativas. Consideraciones finales: Aunque ningún dispositivo tecnológico es capaz de suplir la necesidad de la interacción cara a cara en la educación médica, la monitoria en línea fue fundamental para actuar como facilitador, estableciendo puentes en la interfaz profesor-alumno.
\end{abstract}

Palabras clave: Anestesiología, Educación, Covid-19, Monitoria.

\title{
INTRODUÇÃO
}

Com o surgimento de grave síndrome respiratória aguda iniciada em Wuhan, na China em dezembro de 2019, o mundo passou a enfrentar o medo de uma pandemia. A doença atribuída ao novo coronavírus foi então oficialmente designada de Doença por coronavírus 2019 ou COVID-19 pela Organização Mundial de Saúde (OMS) (KHARASCH ED e JIANG Y, 2020). Com a rápida disseminação mundial do vírus, resposta inflamatória grave, hospitalização e óbitos, houve necessidade de propor o distanciamento social como foma de restringir o número de casos e internações (CRODA JHR e GARCIA LP, 2020; FARIAS HSFD, 2020).

A propagação da doença emergiu em meio a dúvidas sobre a eficácia das vacinas e limitação de acesso após desenvolvimento. O cenário mundial por sua vez expõe o medo e as incertezas sobre as variantes do vírus, enquanto a sociedade brasileira vive ainda o f enômeno mundial do comprometimento do trabalho, da renda e da educação formal (ARRUDA EP, 2020; RODRIGUEZ-MORALES AJ, et al., 2020; SANTOS JAF, 2020; DYER O, 2021; SHIEHZADEGAN S, et al., 2021).

O distanciamento humano também provocou impactos no sistema educacional e o Ensino à Distância $(E a D)$ surge não mais como opção, mas como necessidade na tentativa de mitigar as dificuldades da interrupção das aulas presenciais (MINISTÉRIO DA EDUCAÇÃO, 2020a; GIMENEZ AMN, et al., 2020; JOYE CR, et al., 2020).

Como direito social, a educação esbarra na dificuldade de realizar a formação mesmo daqueles que estão envolvidos como trabalhadores na área da saúde (OLIVEIRA ZM, et al., 2020). No contexto do ensino remoto é imprescindível a reflexão sobre o acesso a um ambiente de aprendizado para todos os alunos (ARRUDA EP, 2020; MEDEIROS JHBD, et al., 2020). Na educação médica, a busca por soluções desafia a capacidade dos alunos e professores no manejo de metodologias de ensino, com uso de Tecnologia da Informação e da Comunicação $(T I C)$, necessária para elaboração de materiais que exigem diferentes graus de conhecimento (LI Q, 2007; WILLIAMSON B, et al., 2020).

O retorno das atividades acadêmicas em educação médica por meio do ensino remoto sofreu o impacto da transmissão comunitária do coronavírus no estado do Rio de Janeiro e acarretou novos desafios ao binômio ensino-aprendizado na universidade (MEDEIROS JHBD, et al., 2020; GIMENEZ AMN, et al., 2020). O ensino da anestesiologia impõe o modelo prático inserido em meio a exposição gradual de tópicos teóricos (CAVALCANTI IL, et al., 2017).

Em resposta à necessidade de rápida adaptação exigida pelo início deste novo modelo, os professores enfrentaram o desafio de adequação ao ensino remoto (HODGES CB, et al., 2020). A presença de monitores neste contexto torna-se essencial na criação de pontes na comunicação entre professores e alunos e no 
desenvolvimento de materiais para a interface online. No módulo de anestesiologia, os monitores têm como escopo a introdução aos conceitos de docência e ao aprofundamento dos conhecimentos, participaram do projeto em evolução, no qual a aprendizagem se daria por meio de ambiente virtual e intervenções pedagógicas e de suporte ao aluno, mediadas por inovações tecnológicas (MEDEIROS MRD, et al., 2020).

Este trabalho tem como objetivo relatar a experiência na implementação do projeto de monitoria online de anestesiologia no sétimo período de uma faculdade de medicina no estado do Rio de Janeiro, o seu desenvolvimento, os resultados iniciais e autoavaliações por professores, alunos e monitores. Objetiva-se ainda pontuar as percepções sobre esta experiência de ensino em anestesiologia e perspectivas de aperf eiçoamento futuro.

\section{RELATO DE EXPERIÊNCIA}

A monitoria em anestesiologia foi administrada remotamente pela plataforma Google Sala de Aula (GSA) para duas turmas consecutivas da faculdade de medicina, entre o segundo semestre de 2020 o primeiro de 2021. Participaram deste processo um total de nove professores, dois monitores e 155 alunos. Os temas trabalhados foram: segurança do paciente, acesso venoso, avaliação e visita pré-anestésica, anestesias venosa e geral, bloqueios centrais, aparelho de anestesia, monitorização, reposição volêmica, intubação orotraqueal e oxigenioterapia, recuperação pós-anestésica, dor e reanimação cardiorrespiratória.

A dinâmica envolveu momentos assíncronos e síncronos, de acordo com a metodologia de aula invertida. Inicialmente havia a postagem de literatura de referência, casos clínicos e vídeos curtos de domínio público ou de arquivo pessoal dos prof essores em ação com procedimentos anestésicos. O momento síncrono ocorria uma semana após a postagem destes materiais e tinha como objetivo a interação online e simultânea entre alunos, professores e monitores para a abordagem teórica dos temas e esclarecimento de dúvidas. Um questionário autoavaliativo foi aplicado aos professores e alunos ao final de cada semestre.

As principais dúvidas levantadas no início da implementação da disciplina e monitoria em ambiente remoto foram sobre o processo avaliativo, prazos de entrega dos trabalhos, dificuldade de acesso nos momentos síncronos, organização de materiais assíncronos, necessidade de padronização das extensões dos arquivos de texto e vídeo e adaptação de conteúdos práticos ao ambiente de ensino remoto.

Houve acordo sobre as estratégias de ensino, levando em consideração as demandas e a realidade particular dos alunos. A avaliação foi padronizada por meio de casos clínicos elaborados com grau de dificuldade e concepção prévia, desenvolvidos a partir da interação entre os alunos durante sua realização. Estes casos eram enviados pelo Google forms pelos monitores com prazo de uma semana para entrega.

Como forma de contribuir para o planejamento e seguimento do cronograma de estudos, os materiais de cada tema passaram a ser postados todos em um único dia da semana, em calendário divulgado previamente. As ferramentas "material" e "atividade" contidas no GSA foram essenciais para que os alunos pudessem visualizar os materiais categorizados, assim como marcar as atividades já concluídas ou não e seus prazos de entrega.

A padronização das extensões dos arquivos foi PDF para textos, mp4 para vídeos e mp3 para arquivos de áudio. Houve a preferência de parte dos professores pela gravação de aulas através de slides co mentados. Dada a dificuldade de abertura de tais arquivos pelos alunos, os monitores transformaram estes arquivos em vídeos e extraíram e unificaram os áudios na criação de podcasts, pensando nos alunos com limitação de cobertura de internet e dados. Vídeos longos foram desencorajados e desmembrados, caso passassem de 30 minutos.

Os encontros síncronos ocorreram pelo Google Meet, disponível dentro da GSA e duravam aproximadamente uma hora. Estes eram gravados e editados pelos monitores, por meio do programa OBS Studio e disponibilizados posteriormente para aqueles que não pudessem participar ao vivo. Em média, somente metade dos alunos compareciam às reuniões síncronas e um quarto interagiam ativamente. No entanto, houve outras formas de participação, como mensagens particulares no GSA ou contatos por redes sociais com os monitores na retirada de dúvidas e exposição de dificuldades. 
As dificuldades tecnológicas dos professores sobre as ferramentas do Google Meet foram resolvidas em reuniões virtuais particulares com os monitores, antes de cada atividade síncrona. Já a necessidade de abordagem prática foi trabalhada com vídeos de procedimentos realizados pelos próprios professores. Os monitores contibuíram na inserção de legendas em português de vídeos originalmente na língua inglesa e atuação como pacientes em simulações da prática médica.

Os questionários semiestruturados aplicados ao final dos períodos trouxeram as percepções de professores e alunos sobre todo o trabalho realizado. Entre os professores, as expectativas pedagógicas foram atendidas plenamente porcinco e parcialmente porquatro. Oito consideraram o ambiente do GSA como propício e estimulante para a aprendizagem. Um professor considerou o tempo dos vídeos assíncronos e das atividades síncronas como totalmente insatisfatório. Os casos clínicos foram considerados satisfatórios para quatro dos cinco professores que empregaram esta metodologia. Somente dois não tiveram nenhuma dificuldade com o uso da tecnologia.

O questionário teve nove perguntas fechadas e quatro abertas e foi respondido por cerca de $25 \%$ dos alunos, de forma voluntária e anônima. As expectativas da disciplina foram atendidas por $78,9 \%$. A participação e o grau de envolvimento foram classificados como satisfatórios por $42,1 \%$ e parcialmente por $52,6 \%$. Cerca de metade dos alunos acreditaram que o aprendizado foi satisfatório e que o ambiente do Google Meet e GSA favoreceu o aprendizado.

O tempo dos vídeos assíncronos foi satisfatório para todos os alunos e 94,7\% ficaram satisf eitos com o tempo de aula síncrona. A maioria dos alunos que respondeu ao questionário informou que não houve dificuldade tecnológica $(89,5 \%)$ ou dificuldade de acesso à internet $(78,9 \%)$ durante o período da disciplina.

Muitos dos alunos elogiaram a qualidade dos encontros síncronos, comprometimento e pontualidade dos professores e ressaltaram a importância e organização da monitoria. Eles classificaram os vídeos das práticas anestésicas como ponto positivo e sugeriram que esta ferramenta fosse mais amplamente utilizada. Por fim, as monitoras consideraram o trabalho como edificante para a formação profissional e para o desenvolvimento de competências e habilidades em anestesiologia.

\section{DISCUSSÃO}

As universidades públicas federais tiveram papel relevante no combate à pandemia por Covid -19 . Suas ações envolveram produção de conhecimento científ ico, de equipamentos de proteção individual e materiais educativos para a população, apoio psicológico à sociedade, concessão de espaços para instalação de hospitais de campanha e atuação de hospitais no atendimento assistencial aos pacientes com Covid -19 (GIMENEZ AMN, et al., 2020; SALVADOR PTCO, et al., 2020).

A suspensão das aulas presenciais, apesar de gerar ansiedade e angústia em toda a comunidade acadêmica, foi necessária para diminuir a disseminação do coronavírus, considerando a sua transmissão comunitária em território nacional. Em curto espaço de tempo foi preciso planejar medidas para instituir atividades educacionais e administrativas remotas emergenciais (MAIA BR e DIAS PC, 2020; MEDEIROS JHBD, et al., 2020). As universidades públicas levaram em média seis meses para implementação do ensino em modo remoto (MINISTÉRIO DA EDUCAÇÃO, 2020a; MINISTÉRIO DA EDUCAÇÃO, 2020b).

Para esta implementação foram necessários planejamento e pesquisa sobre perfil e limitações de acesso e conhecimentos tecnológicos de discentes e docentes (ARRUDA EP, et al., 2020; GIMENEZ AMN, et al., 2020). Mesmo que ocorram adaptações na entrega de conteúdos curriculares, ainda assim deve haver o desenvolvimento a médio e longo prazo de estratégias de ensino e aprendizagem sobre as particularidades do ensino remoto, ainda mais considerando cenários pandêmicos de surgimento de novas variantes que acarretem a persistência deste modelo de ensino (ARRUDA EP, 2020; HODGES CB, et al., 2020; DYER O, 2021; SHIEHZADEGAN S, 2021).

Há necessidade de diferenciar Ensino à Distância $(\mathrm{EaD})$ classicamente estabelecido de atividades remotas emergenciais. O EaD se constitui como modalidade com tempo de implementação e pesquisa consolidada e que conta com investimento e profissionais da área tecnológ ica como designers educacionais e revisores, 
além de professores capacitados previamente. Nas atividades educacionais remotas emergenciais, 0 professor trabalha muitas vezes de forma isolada, sem auxílio de outros profissionais e sem capacitação tecnológica estruturada prévia e o conteúdo é elaborado de forma totalmente remota (HODGES CB, et al., 2020; JOYE CR, et al., 2020).

A figura do monitor aparece com grande potencialidade ao construir pontes entre professores e alunos, auxiliar na interface do uso dos dispositivos TICs e elaborar materiais didáticos e práticas que minimizem os prejuízos da falta de aulas presenciais. Padronização dos arquivos enviados aos alunos, criação de podcasts, gravação e edição de vídeos da prática médica e adição de legendas são alguns exemplos de ações da monitoria. Silva M, et al. (2020) e Sadigurschi G, et al. (2021) exemplificam outras ações dos monitores, como a construção de mapas mentais, o estudo de imagens, exames e casos clínicos.

A sala de aula virtual do GSA, utilizada neste trabalho, existe como ferramenta segura e de fácil manejo pelo educador, que busca gerenciar o tempo e tornar mais interessante a experiência de ensinoaprendizagem. Tal organização permite administrar numa só plataforma diferentes turmas, que podem trabalhar conteúdos temáticos. $\mathrm{O}$ acesso ocorre a partir de qualquer lugar, assim há garantia de mobilidade e emprego de recursos, como atividades, tarefas e testes com maior flexibilidade (MARTINS J, et al., 2019). O GSA também auxiliou aos alunos na organização de estudo ao discriminar os materiais e atividades já estudadas ou realizadas e confrontar o limite de prazos estabelecidos para cada tarefa.

Muitos professores apresentaram dificuldade na utilização de ferramentas digitais e tiveram o auxílio das monitoras nas atividades síncronas e assíncronas. Houve grande heterogeneidade no conhecimento tecnológico entre os professores das plataformas utilizadas, o que gerou receio de não transmitir os conhecimentos necessários. Para Barros LCMD, et al. (2022), houve difícil adaptação dos docentes às ferramentas tecnológicas do ensino remoto no contexto pandêmico, sendo crucial um treinamento institucional para a adaptação às metodologias. $\mathrm{Na}$ verdade, a presença de monitores aliada a maior treinamento institucional aumentam a perspectiva de melhoria na eficácia deste novo instrumental.

Como limitação podemos apontar a restrição observada a um grupo de alunos com dificuldade de acesso à internet, o que pode conferir viés de seleção (amostragem) na avaliação do questionário aplicado e estimativa tendenciosa a inquéritos. A subcobertura representa problema, que foi trabalhado com áudios gravados relacionados ao material síncrono, como na resolução dos casos clínicos, as sim permitindo melhor abrangência a todo corpo discente. No ambiente virtual, no entanto, a motivação pelo novo instrumento de ensino-aprendizagem e a falta de obrigatoriedade do acesso não foram objetos de análise (BETHLEHEM J, 2010).

É esperado que o contexto tecnológico seja encarado com naturalidade pelos alunos e a experiência como positiva. A menor participação deles no uso da plataforma pode representar falta de motivação em tempos de pandemia, mas também aponta para necessidade de melhoria no processo avaliatório remoto. Prazos e conteúdos podem ser mais flexíveis, aulas com simulações de situações clínicas melhoradas. Diante da necessidade prática da interação, cuja busca tem perfil dinâmico, o ambiente virtual contribuiu na superação do momento desafiador, mas precisa evoluir como instrumento que melhore o ensino.

A implementação do projeto de monitoria auxiliou o ensino de anestesiologia no ambiente remoto. Os resultados iniciais foram satisfatórios quanto ao emprego de ferramentas tecnológicas, que podem ainda ser aprimoradas. As autoavaliações revelaram as dificuldades encontradas no uso de TICs, mas com o mérito do desenvolvimento de soluções pedagógicas inovadoras. A experiência valorizou a interface docente-discente, com importante mediação realizada pelos monitores, assim como o trabalho permitiu a descrição de alternativa original do ensino remoto para a especialidade.

\section{REFERÊNCIAS}

1. ARRUDA EP. Educação remota emergencial: elementos para políticas públicas na educação brasileira em tempos de Covid-19. Rede-Revista de Educação à Distância, 2020; 7(1): 257-275.

2. BARROS LCMD, et al. Percepção dos docentes sobre o ensino remoto em medicina durante a pandemia pela COVID19. Research Society and Development, 2022;11(1):e52411125205-e52411125205. 
3. BETHLEHEM J. Selection bias in web surveys. International Statistical Review, 2010;78(2): 161 -188.

4. CAVALCANTIIL, et al. O ensino de anestesiologia na graduação. In: James Manica. (Org.). Anestesiologia. 4ed. Porto Alegre: Artmed, 2017; 1:63-68.

5. CRODA JHR, GARCIA LP. Immediate Health Surveillance Response to COVID-19 Epidemic. Epidemiol Serv Saude, 2020;29(1): e2020002.

6. DYER O. Covid-19: South Africa's surge in cases deepens alarm over omicron variant. 2021. BMJ, 2021; 375(3013): 1-2.

7. FARIAS HSFD. O avanço da Covid-19 e o isolamento social como estratégia para redução da vulnerabilidade. Espaço e Economia:Revista Brasileira de Geografia e Economia, 2020;17:1-12.

8. GIMENEZ AMN, et al. Universidades Brasileiras e Covid-19: fortalecendo os laços com a sociedade. Boletim Unicamp, 2020;1-4.

9. HODGES CB, et al. The difference between emergency remote teaching and online learning. EDUCAUSE Review 2020;1-12.

10. JOYE CR, et al. Distance Education or Emergency Remote Educational Activity: in search of the missing link of school education in times of COVID-19. Research, Society and Development, 2020; 9(7): e521974299.

11. KHARASCH ED, JIANG Y. Novel coronavirus 2019 and anesthesiology. Anesthesiology, 2020; 132(6):1289-1291.

12. LI Q. Student and teacher views about technology: A tale of two cities? Journal of Research on Technology in Education, 2007;39(4): 377-397.

13. MAIA BR, DIAS PC. Ansiedade, depressão e estresse em estudantes universitários: o impacto da Covid-19. Estudos de Psicologia, 2020;37:e200067.

14. MARTINS J, etal. Avaliação do Google Sala de Aula como Ferramenta de Apoio ao processo de ensino-aprendizagem em um curso de licenciatura em Ciências Biológicas presencial. Renote, 2019;17(3): 587-596.

15. MEDEIROS JHBD, et al. Desafios do ensino superior durante a pandemia do Covid-19: relato de experiência da monitoria de anatomia humana. Braz Journal of Development, 2020;6(10):84008-84015.

16. MEDEIROS MRD, et al. Papel da monitoria na formação acadêmica em tempos de COVID -19: Relato de Experiência. Revista Extensão e Sociedade, 2020;12(1):120-129.

17. MINISTÉRIO DA EDUCAÇÃO. Associação Brasileira de Mantenedoras de Ensino Superior. Gabinete do Ministro. Portaria no 343, de 17 de março de 2020a. Dispõe sobre a substituição das aulas presenciais por aulas em meios digitais enquanto durar a situação de pandemia do Novo Coronavírus - COVID-19. Diário Oficial da União, Brasília, DF, ed. 53, 18 mar. 2020. Seção 01, p. 39. Disponível em: https://abmes.org.br/arquivos/legislacoes/Portaria-mec-3432020-03-17.pdf. Acesso em: Acessado em: 03 de janeiro de 2022.

18. MINISTÉRIO DA EDUCAÇÃO. Gabinete do Ministro. Portaria ํㅜ 544, de 16 de junho de 2020b. Dispõe sobre a substituição das aulas presenciais por aulas em meios digitais, enquanto durar a situação de pandemia do novo coronavírus - Covid-19, e revoga as Portarias MEC ํㅡ 343, de 17 de março de 2020, № 345, de 19 de março de 2020, e $\mathrm{n}^{\circ} 473$, de 12 de maio de 2020 . Disponível em: https://pesquisa.in.gov.br/imprensa/jsp/visualiza/index.jsp?data=17/06/2020\&jornal=515\&pagina=62. Acessado em: 03 de janeiro de 2022.

19. OLIVEIRA ZM, et al. Estratégias para retomada do ensino superior em saúde frente a COVID-19. Revista Enfermagem Atual In Derme, 2020;93: 1-21.

20. RODRIGUEZ-MORALES AJ, et al. COVID-19 in Latin America:The implications of the first confirmed case in Brazil. Travel Medicine and Infectious Disease, 2020;35: e101613.

21. SADIGURSCHI G, et al. Experiência dos monitores da disciplina de hematologia e hemoterapia da unirio durante o período de pandemia de covid-19. Hematology, Transfusion and Cell Therapy, 2021;43: S503.

22. SALVADOR PTCO, et al. Ações e repercussões das primeiras iniciativas das universidades públicas federais brasileiras frente à COVID-19. Holos, 2020;5:1-17.

23. SANTOS JAF. Covid-19, causas fundamentais, classe social e território. Trabalho, Educação e Saúde, 2020; 18(3): 17.

24. SHIEHZADEGAN S, et al. Analysis of the delta variantB. 1.617.2 COVID-19. Clinics and Practice, 2021; 11(4): 778784.

25. SILVA M, et al. Monitoria de ensino e atividades remota durante a pandemia do coronavírus na disciplina vigilância em saúde: relato de experiência. . Revista Multidisciplinar do Núcleo de Pesquisa e Extensão (RevNUPE), 2021;1(1): e202112-e202112.

26. WILLIAMSON B, et al. Pandemic politics, pedagogies and practices: digital technologies and distance education during the coronavirus emergency. Learning, Media and Technology, 2020;107-114. 